\title{
versants
}

\section{Studiare le carte dell'Archivio Prezzolini: ipotesi di lavoro e casi di studio}

Giulia Pellizzato

Brown University - Università della Svizzera italiana

\begin{abstract}
L'Archivio Prezzolini custodisce un'ampia messe di documenti, rilevanti sotto molteplici punti di vista. Dopo una presentazione dei fondi letterari più significativi dell'Archivio, ci si concentrerà sulle carte di Prezzolini. Fra queste vi sono documenti ancora da studiare che, oltre a testimoniare l'eccezionale attività culturale del possessore, si rivelano preziosi per ricerche dedicate a tematiche più generali e altri autori. La seconda parte dell'articolo mostrerà il potenziale di tali documenti illustrando l'attività culturale di Prezzolini come mediatore fra Italia e Stati Uniti.
\end{abstract}

Keywords: Giuseppe Prezzolini, Archivio Prezzolini, filologia del Novecento, carteggi, relazioni culturali.

Con i suoi ottocento metri lineari di documenti l'Archivio Prezzolini, conservato presso la Biblioteca cantonale di Lugano, è fra i maggiori archivi letterari del Novecento in lingua italiana. Sui fondi che lo compongono sono in corso svariate ricerche, tracciabili grazie alla sezione «Lavori in corso» di Cartevive, il periodico dell'Archivio. La stessa rivista offre un censimento delle pubblicazioni basate su documenti appartenenti all'Archivio, segnala le nuove accessioni di documenti, riporta approfondimenti su temi e documenti relativi e registra le attività dell'Archivio stesso dal 1990 a oggi, costituendo un utile strumento di lavoro per chi voglia avvicinarsi allo studio delle sue carte ${ }^{\mathrm{I}}$.

Il primo nucleo di manoscritti dell'Archivio si costituì oltre quarant'anni fa, quando Prezzolini ipse cedeva duecento scatole di «lettere, cartoline, opuscoli, ritagli di giornale, fotografie [...] raccolte e trasportate per ben 76 anni attraverso Italia, Francia, Germania, Stati Uniti, Italia di nuovo» (Prezzolini 1983: 23) alla Biblioteca cantonale di Lugano. Era il 1978. Nel corso degli anni al Fondo Prezzolini, composto da circa trentamila pezzi, si sono aggiunti numerosi Fondi e Raccolte: fra questi sarà opportuno ricordare almeno le carte di Francesco Chiesa, Ennio Flaiano, Guido Calgari, Guido Ceronetti, Enrico Emanuelli, Giovanni Battista Angioletti, Fulvio Tomizza, Alessandro Spina - seguendo l'ordine cronologico di entrata.

I Cartevive. Periodico dell'Archivio Prezzolini, Biblioteca cantonale di Lugano, 1990-2019. Ogni volume verrà indicato con la numerazione che compare sul frontespizio perché il sistema di numerazione è cambiato nel corso degli anni. 
Larchivio del poeta, scrittore ed educatore ticinese Francesco Chiesa comprende carteggi con centinaia di destinatari, oltre agli scritti editi (libri, opuscoli e articoli), ai ritagli di stampa, ai progetti di legge e ad alcuni fascicoli legati alla politica del Canton Ticino. Nel 2004 l'Archivio Prezzolini ha acquisito il Fondo de Haller-Chiesa, catalogato, che comprende manoscritti, corrispondenze e biblioteca dell'autore (quest'ultima giunta a Lugano nel 20I7). Il Fondo de Haller-Chiesa, parte integrante del Fondo Chiesa, è già stato oggetto di studi sfociati in opere edite ${ }^{2}$.

Il Fondo Flaiano, inventariato e oggetto di alcune pubblicazioni (fra cui i cataloghi delle mostre dedicate alle carte che lo compongono) ${ }^{3}$, comprende l'epistolario, l'emeroteca e la biblioteca di studio dell'autore (quest'ultima consultabile unicamente in sede, come ogni biblioteca d'autore, presso la Biblioteca cantonale di Lugano), più altre sezioni, per un totale di tredici. Fra queste si segnalano quella contenente materiale iconografico, con disegni di Francesco Ciarletta, Federico Fellini, Mino Maccari, oltre a fotografie scattate nell'arco di settant'anni, e quella dedicata al materiale cinematografico, con oltre cinquanta sceneggiature di film (realizzati e no), una trentina di soggetti, oltre a giudizi e interventi critici dell'autore, e corrispondenze con produttori, registi e attori (cfr. Rüesch 1990; Flaiano 1990).

Larchivio di Calgari, raccolto in dieci scatole, è stato inventariato dalla figlia, Fiorenza Calgari Intra, donatrice delle carte paterne all'Archivio Prezzolini ${ }^{4}$. Vi si trovano i materiali pubblicati, in periodici e in volume (con raccolte d'autore e postume), i documenti relativi a interventi radiofonici di varia natura, una sezione dedicata a «Calgari tradotto». La maggior parte degli articoli è semplicemente elencata: i testi cartacei sono da cercare soprattutto tra le riviste e i giornali della Libreria Patria (Biblioteca cantonale di Lugano).

Guido Ceronetti invece ha ceduto le proprie carte all'Archivio Prezzolini in prima persona, a più riprese; oggi il suo archivio è uno dei fondi più cospicui, con una raccolta che sfiora il centinaio di scatole. Nella sezione «Opera edita e inedita» si trovano più di quattrocento quaderni autografi, la «Corrispondenza» ammonta a svariate migliaia di esemplari. Vi sono poi alcune sezioni iconografiche. Dal Fondo Ceronetti sono stati tratti varie mostre ${ }^{5} \mathrm{e}$ diversi volumi (Ceronetti 2000, 2016, 2017; Ceronetti-Quinzio 2014).

2 Cfr. ad esempio Chiesa-Formiggini 2010.

3 Ferrario-Rüesch 1988; Flaiano 200I, 2002 (catalogo della mostra tenuta a Perugia fra il 2002 e il 2003), 2004, 2007, 2010. Cfr. inoltre il catalogo della mostra tenuta a Lugano nel 2010: Rüesch-Stefanski 20ıo. L'opera letteraria di Flaiano è conservata presso il Fondo Manoscritti di Autori Moderni e Contemporanei dell'Università di Pavia.

4 Cfr. Calgari Intra 1990; https://www.sbt.ti.ch/bclu/archivio/pdf/InvFondoCalgari.pdf (consultato il I5 gennaio 2019).

5 Ricordo la prima e l'ultima: Rüesch-Franciolli 2000; Rüesch-Stefanski 2017. 
Il Fondo Enrico Emanuelli, già forte di una documentazione consistente, si è ulteriormente arricchito nel corso del 2018 grazie a una significativa accessione di nuovi materiali, attualmente in corso di riordino. Le carte donate precedentemente all'Archivio Prezzolini comprendono racconti e articoli giornalistici (manoscritti e dattiloscritti), l'epistolario dell'autore, ritagli di stampa.

Il Fondo Angioletti si distingue per la nutrita raccolta di articoli a stampa, dell'autore e sull'autore. A questa si aggiungono una cospicua sezione fotografica e un ampio epistolario (Bonini-Callegari Maestretti-Rüesch 2003; cfr. inoltre Saltini 2007), svariati manoscritti (quaderni con appunti e progetti di libri, prime stesure di racconti e prose editi) e testi editi.

Le carte di Fulvio Tomizza, raccolte in oltre sessanta scatole, comprendono manoscritti e dattiloscritti relativi all'intera attività dell'autore: racconti e romanzi editi (per ciascuno dei quali è presente una rassegna critica) e ancora testi per il teatro e per trasmissioni radiofoniche. Vi sono inoltre appunti, studi, abbozzi e progetti per opere narrative, saggistiche e giornalistiche. Il fondo raccoglie l'intero archivio dell'autore, a cui è stata dedicata una mostra presso la Biblioteca cantonale di Lugano ${ }^{6}$.

Il Fondo Alessandro Spina (nom de plume di Basili Khouzam) comprende i manoscritti dei volumi raccolti nell'edizione I confini dell'ombra (Brescia, Morcelliana, 2007): undici testi fra romanzi e raccolte di racconti, dedicati all'esperienza coloniale italiana in Africa. Vi è poi l'interessante corrispondenza con poeti e scrittori, prima fra tutte quella con Cristina Campo, di cui Spina curò l'edizione per i tipi di Scheiwiller (Campo 1989)7, ma anche quelle con Anna Banti, Giorgio Bassani, Pietro Citati, Alberto Moravia, Giovanni Orelli, Sergio Solmi, Elémire Zolla (cfr. Rüesch 200I).

Schede dettagliate su questi e altri Fondi possono essere consultate in rete: l'Archivio Prezzolini ammonta oggi a settantatré nuclei archivistici, ovvero trenta Fondi e quarantatré Raccolte di minore entità. Fra questi, il Fondo Giuseppe Prezzolini è indubbiamente il maggiore. Alle carte acquistate nel 1978, cui si accennava all'inizio, si sono aggiunti i documenti donati a più riprese dal figlio Giuliano Prezzolini, che comprendono lettere (a Prezzolini e alla prima moglie, Dolores Faconti), libri (spesso impreziositi da dediche manoscritte degli autori), fotografie (cfr. Cucchiaro 1996), mentre

6 Da Materada a Materada. Un viaggio nella creazione letteraria di Fulvio Tomizza dagli esordi a La miglior vita (Lugano, 24 ottobre - I4 novembre 2009). Cfr. Stefanski 2009.

7 A questa seguì un'edizione comprensiva delle repliche di Spina: Campo-Spina 2007.

8 Cfr. https://www.sbt.ti.ch/bclu/?m=archivio (consultato il I5 gennaio 2019). Allo stesso indirizzo sono disponibili, tra gli altri, gli inventari dei Fondi Calgari e Valagnin, e della corrispondenza dei Fondi Angioletti e Felice Filippini. 
decine di acquisizioni di altra provenienza hanno integrato i carteggi già archiviati ${ }^{9}$.

La responsabile Diana Rüesch ha presentato sistematicamente le carte che compongono il Fondo Prezzolini in più occasioni ${ }^{10}$; in questa sede mi soffermerò quindi su documenti e temi d'interesse in vista di possibili ricerche future.

Si potrà iniziare accennando agli innumerevoli ritagli di giornale che compongono tre sezioni: «Raccolta degli articoli di Prezzolini», «Raccolta di recensioni alle opere di Prezzolini», «Raccolta di articoli su Prezzolini e l'ambiente vociano». Riguardo alla prima, il lavoro di Prezzolini negli anni del Leonardo e della Voce è stato oggetto d'interesse e di studio sin dagli anni Cinquanta, mentre la successiva attività giornalistica, tenuta su numerose testate italiane e straniere, è stata esplorata con minore frequenza. Le ricerche che ho compiuto per il mio lavoro di dottorato (Pellizzato 20I8), incentrato sul carteggio fra Prezzolini e Goffredo Parise, hanno mostrato che fra il I95I e il I976 - l'intero arco di anni interessato dal carteggio - Prezzolini scrisse al ritmo di almeno due o tre articoli a settimana. Le collaborazioni regolari spesso uscivano in parallelo su diverse testate: Il Resto del Carlino, La Nazione, Il Tempo e più tardi Il Giornale d'Italia. Gli articoli redatti per Il Borghese, invece, uscivano solo sul giornale di Longanesi. Vi è poi una miriade di contributi saltuari consegnati ad altri periodici, italiani e americani, che attende uno studio sistematico ${ }^{\mathrm{II}}$.

La ricerca sul corpus degli articoli di Prezzolini potrà presto avvalersi di una bibliografia a stampa, attualmente in lavorazione presso l'Archivio a cura di Diana Rüesch e Karin Stefanski, mentre è disponibile in sede lo schedario cartaceo, arricchito nel corso degli anni anche grazie alle segnalazioni di vari studiosi. A questo corrisponde una nutrita raccolta di ritagli, inizialmente curata da Prezzolini stesso. Si tratta di una produzione straordinaria per estensione testuale, ampiezza e originalità d'interessi, che offre materia d'indagine da svariate prospettive di ricerca. Prezzolini fu un attento osservatore degli Stati Uniti e degli equilibri geopolitici internazio-

9 Le nuove accessioni sono registrate nella sezione «Nuove Accessioni» di Cartevive. Alle carte di Prezzolini sono state dedicate varie esposizioni, cfr. Pino Pongolini 1982; Rüesch 1997. Io Cfr. Rüesch 200I, 2003, 2005. Cfr. inoltre Pongolini-Rüesch 1989; https://www.sbt.ti.ch/ dep/bclu/l_archivio_prezzolini.pdf (consultato il I5 gennaio 2019).

II Fra le testate italiane: i settimanali milanesi L'Europeo, L'Illustrazione Italiana e Epoca, Candido, i settimanali romani Idea, L'Osservatore della Domenica e L'Elefante, i periodici di argomento letterario Il Ponte e La Fiera Letteraria, e poi ancora il quotidiano Paese Sera, La Nuova Stampa, il settimanale La Destra, Video, Lo Spettacolo, Tuttitalia, Terra di Siena, I problemi della Pedagogia. Alle collaborazioni già note è da aggiungere quella con Cronache della politica e del costume, settimanale antesignano di L'Espresso, che ho individuato nel corso di ricerche presso l'emeroteca dell'Archivio Storico Capitolino a Roma. Fra le testate americane: il New York Times, Atlas, Italamerican, Italian Cultural Institute, Cesare Barbieri Courier. 
nali, scrisse sull'Italia contemporanea da oltreoceano (dagli anni Trenta ai Cinquanta), dall'interno e più tardi dalla Svizzera (dagli anni Sessanta agli Ottanta), fu un sociologo di lungimiranza penetrante, registrò con disincanto le trasformazioni culturali in atto, nelle istituzioni scolastiche come nel sistema letterario, intervenne da critico militante sui libri editi sia in italiano sia in altre lingue, segnalando al pubblico testi che altrimenti sarebbero rimasti ignoti più a lungo. I suoi scritti giornalistici offrono materiale di notevole interesse, sia per lo studio del pensiero dell'autore, sia per ricerche storiche e culturali di più ampio respiro.

Un altro possibile cantiere di lavoro riguarda la biblioteca di Prezzolini: oltre tremila volumi, custoditi nei magazzini della Biblioteca cantonale di Lugano e dell'Archivio Prezzolini ${ }^{12}$. Si tratta di libri doppiamente rilevanti: in primo luogo testimoniano le eclettiche letture del possessore, non ancora oggetto di studio scientifico per quanto riguarda il periodo successivo alla gioventù ${ }^{13}$. Un confronto sistematico fra scritti e postille di lettura permetterebbe di approfondire gli studi sulla sua cultura letteraria, storica, politica, ma anche scientifica. In merito a quest'ultimo aspetto, uno studio che ho avviato intorno alla ricezione delle scoperte scientifiche negli ambienti letterari italiani sta mostrando che Prezzolini s'interessava di biologia già nel corso degli anni Venti, e che tali letture probabilmente influenzarono le posizioni filosofiche e politiche da lui assunte nella seconda metà del secolo. In secondo luogo, quando latori di dediche o postille, i volumi di Prezzolini contengono informazioni e testimonianze che offrirebbero indicazioni preziose, se intrecciate agli altri dati offerti dalle carte d'archivio. Nel caso della già menzionata relazione fra Prezzolini e Parise, ad esempio, le dediche rinvenute sui libri di Prezzolini hanno permesso di documentare e precisare cronologicamente alcuni periodi non rappresentati dalle lettere rinvenute sino a oggi.

Fra i documenti presenti in Archivio non si potranno certamente trascurare i carteggi, siti nelle sezioni «Corrispondenze antiche» $\mathrm{e}$ «Corrispondenze moderne». Dell'ottuagenaria attività di Prezzolini, «impresario di cultura» (Simonetti I98I; Cadioli I995: 68) sin dai tempi della Voce, «uomo pratico» (Prezzolini 1953: 349), e ancora «uomo-ponte» (Prezzolini I958: 7) fra Italia e Stati Uniti, poliedrico scrittore in proprio, studioso, professore universitario e prolifico giornalista, rimangono scambi epistolari con oltre tremila e settecento interlocutori ${ }^{14}$. A fronte di un simile numero sarà sorprendente

I2 Volumi rintracciabili sotto le sigle: LGC 072 Pr; LGC 073 Pr; LGC o74 Pr.

I3 Sino a oggi, in effetti, gli studi sul pensiero di Prezzolini si sono concentrati sulla sua formazione intellettuale: cfr. Campanile 1985; Salek 2002; Biondi 2005. I volumi della biblioteca luganese offrono quindi informazioni sul periodo meno noto degli studi di Prezzolini. Cfr. inoltre Gentile 1994.

I4 Cfr. Rüesch 200I, 2003, 2005, https:/www.sbt.ti.ch/dep/bclu/1_archivio_prezzolini.pdf 
constatare come i carteggi conservati siano solo una parte di quelli effettivamente intrattenuti dall'autore. Ciò si deve ad almeno due fattori. In primo luogo gli agenti esterni, come racconta Prezzolini stesso nella prefazione al primo volume che trasse dal proprio archivio epistolare, Il tempo della Voce:

Son state trasportate [le lettere] di qui e di là per il mondo, da una soffitta di Firenze a una cantina di Roma, parte in un sottosuolo di un dotto e letterato sacerdote che abitava vicino al Colosseo, parte in un principesco palazzo di Parigi, poi in una località allora deserta presso il Forte dei Marmi [...]. Quando stavan riposando, credendo d'esser al sicuro dalla tempesta mondiale di quel tempo, si trovaron proprio sulla Linea Gotica, e il tetto dell'edificio che le proteggeva ricevette il biglietto di visita di una bomba, non si sa se dovuta alle intenzioni degli oppressori o dei liberatori, e rimasero esposte all'umidità. La pioggia non badava se guastasse scritture di genii o fatture di tipografi (I960: I3).

In secondo luogo gli effetti di una decurtazione meno appariscente, che pare frutto di una selezione volontaria. Prezzolini visse negli Stati Uniti per oltre trent'anni, dal 1930 fino al I962, quando si trasferì a Ravello con la seconda moglie, Gioconda (Jakie) Savini. Lo stato attuale delle carte sembra suggerire che alla vigilia del rientro in Italia Prezzolini abbia operato una scelta nel proprio ingente archivio, tralasciando le carte che riteneva meno importanti. La prefazione a un libro che preparava proprio in quel periodo (i due volumi del carteggio con l'amico Papini, Storia di un'amicizia) sembra esplicitare il criterio che avrebbe guidato quella cernita: nella prefazione Prezzolini avvertiva che le «faccende pratiche come libri letti o da leggere, somme pagate o da pagare, abbonati possibili o reali alle nostre riviste e via dicendo» venivano tralasciate, in quanto «cosette che potrebbero aver interesse per una storia economica e culturale dei nostri tempi, ma che non si riferiscono direttamente al movimento dei nostri spiriti» (I966: 9; corsivo mio). Tornando al Fondo Prezzolini, se si esaminano i carteggi iniziati dopo la Seconda Guerra Mondiale si può notare che, in effetti, dei contatti editoriali precedenti al trasferimento in Italia non sono rimaste lettere, a meno che non si trattasse di accordi editoriali - da conservare, dunque, per necessità pratiche ${ }^{15}$. Le comunicazioni successive al trasferimento in Europa, invece, risultano presenti. Alla luce delle testimonianze citate e di quanto reperibile fra i carteggi di Prezzolini sembra dunque plausibile che gli scambi epistolari con le case editrici fossero stati eliminati alla vigilia del

(consultato il I5 gennaio 2019).

I5 È questo il caso del carteggio con gli editori Abelard-Schuman e Farrar Straus, presso cui uscirono libri di Prezzolini in traduzione (1955, 1967). Lo stesso si può dire per i carteggi con editori italiani, come Vallecchi, Garzanti, Longanesi, o con figure come Giovanni Spadolini e Giancarlo Vigorelli, lacunosi per le annate precedenti al rientro in Europa. 
ritorno dagli Stati Uniti, perché ritenuti di scarso interesse. Per uno studio circostanziato delle relazioni fra Prezzolini e il sistema culturale statunitense sarà dunque opportuno completare le informazioni disponibili presso l'Archivio Prezzolini con documenti conservati in altri archivi.

Ciò nonostante i carteggi del Fondo luganese sono tanti e tali da costituire una miniera di informazioni e testi, che può essere attraversata anche in direzioni nuove. Fra gli interlocutori epistolari di Prezzolini c'erano scrittori e intellettuali, editori e istituzioni culturali, ma anche un largo numero di lettori: come testimoniano le sue repliche pubbliche, Prezzolini era molto seguíto e spesso direttamente interpellato per lettera. Meno noto forse è che rispondeva alle lettere anche privatamente, cercando di inviare una replica a ciascun lettore che lo aveva contattato. Secondo la testimonianza di Emilio Gentile, Prezzolini si dedicava alla corrispondenza di sera, dopo aver riservato il mattino alla scrittura e il pomeriggio allo studio. Ogni giorno redigeva decine di lettere, anche trenta o quaranta alla volta. Sebbene difficoltoso per la probabile dispersione delle sue lettere, uno studio della corrispondenza con i suoi lettori costituirebbe un'analisi di indubbio interesse per osservare direttamente che interazione potesse esserci fra un intellettuale e il suo pubblico, al di là di quanto appariva sui giornali (ovvero articoli, lettere dei lettori nella sezione corrispondenza e repliche pubbliche).

Tornando all'attività di Prezzolini come ponte fra le due sponde dell'Atlantico gli studi effettuati per la mia tesi di dottorato hanno mostrato che attraverso i decenni, anche dopo la conclusione della sua attività presso Columbia University (di cui si parlerà fra breve), Prezzolini fu il tramite fra autori o editori italiani ed editori statunitensi, in più occasioni e in modi disparati. Combinando i dati offerti dalle carte del Fondo Prezzolini con studi in altri archivi (Fondo Neri Pozza e Archivio Scrittori Vicentini presso la Biblioteca Civica Bertoliana a Vicenza, Fondo Farrar Straus \& Giroux presso la New York Public Library) è stato possibile accertare che Prezzolini si imbatté nel primo libro di Parise piuttosto casualmente, quando l'editore newyorkese Farrar Straus \& Young (al tempo Farrar Straus \& Giroux) stava considerando la possibilità di tradurlo in American English ${ }^{16}$. Trovando il libro di vivo interesse, Prezzolini agì di propria iniziativa per contattare il giovane autore e auspicò la traduzione del libro presso i consulenti della casa editrice. In seguito, quando la Farrar Straus non si mostrò intenzionata a pubblicare il secondo romanzo di Parise in America, Prezzolini propose il nuovo lavoro a Blanche Knopf, presidente della più prestigiosa casa editrice degli Stati Uniti, Alfred A. Knopf Inc. Grazie a questi contatti fu proprio questo editore a pubblicare il terzo romanzo di Parise, Il prete bello, clamo-

I6 Nel merito, cfr. Pellizzato 2016. 
roso successo in Italia e tradotto in sei lingue nel giro di un anno (cfr. Parise 200I: I58I-I588).

Ma l'attività di Prezzolini seguiva anche altre vie: nel corso degli stessi anni egli era in contatto anche con il primo editore di Parise, Neri Pozza, per tramite del comune amico poeta Antonio Barolini, e si offriva come agente letterario per alcuni libri da lui pubblicati. Sarà proprio Pozza a spiegare al proprio autore la particolare natura dell'attività di scouting condotta dall'anziano scrittore: «Badi che Prezzolini, quando si offre, si offre gratuitamente; non è, lo dico tra noi [...], persona che si dà per lucro» ${ }^{17}$. Contrariamente agli agenti letterari di professione, Prezzolini offriva la propria rete di relazioni ad autori e libri che riteneva rilevanti sul piano letterario, oltre che, naturalmente, appetibili per il pubblico statunitense ${ }^{18}$. Avrebbe continuato a esercitare la sua attività di consigliere anche in seguito, una volta rientrato in Europa. Negli anni Sessanta, come testimoniano le lettere del Fondo, Prezzolini era regolarmente in contatto con la Farrar Straus per le traduzioni dei propri libri. Ciò avveniva grazie alla mediazione di Gioconda Prezzolini, consulente e traduttrice per John Farrar e Roger Straus sin dai primi anni Cinquanta, segretaria della Casa Italiana di Columbia per oltre trent'anni, dal 1962 seconda moglie di Prezzolini e da ben prima sua preziosa collaboratrice (Ragusa I994). Di anno in anno Giuseppe e Gioconda Prezzolini segnalavano quelli che ritenevano i libri migliori dell'anno o i più adatti a una riedizione ${ }^{\mathrm{Ig}}$.

A partire dagli anni Cinquanta, dunque, Prezzolini si occupò per lo più di trasmettere informazioni dall'ambiente italiano a quello statunitense, facendo da tramite affinché scrittori, editori o traduttori italiani entrassero in contatto con il sistema librario americano. Nel ventennio precedente si era invece dedicato al flusso di notizie opposto, creando occasioni e strumenti affinché editori, giornalisti, librai e lettori statunitensi potessero conoscere gli scrittori e i libri italiani. Questo cambio di direzione si deve agli incarichi differenti cui Prezzolini assolse in quel giro d'anni: fra gli anni Trenta e gli anni Quaranta ebbe un ruolo istituzionale, riconosciuto e direttamente collegato al sistema culturale statunitense, in quanto direttore (I930-I940) e poi docente (I940-I950) alla Casa Italiana di Columbia University. Studi effettuati sui documenti oggi nel Fondo Prezzolini hanno permesso di ricostruire alcune delle iniziative intraprese in quel periodo.

I7 Lettera di Neri Pozza ad Alberto Denti di Pirajno, datata I aprile 1953. Fondo Neri Pozza, Serie «Corrispondenze Autori», Fascicolo «Denti di Pirajno» (Biblioteca Civica Bertoliana di Vicenza).

I8 È questo il caso di Quaretti 1947 e Denti di Pirajno 1952, come ho documentato in Pellizzato 2018.

I9 Lo testimoniano le lettere conservate nel Fondo Prezzolini, Serie «Corrispondenze Moderne», Fascicolo «Farrar Straus». 
Come ha raccontato Olga Ragusa, nel corso degli anni Trenta Prezzolini fu attivo come intermediario negli Stati Uniti per la cultura e la letteratura d'Italia attraverso strumenti disparati. Per far fronte alle numerose richieste d'informazioni che sollecitavano la Casa Italiana, concepì e realizzò uno schedario di autori italiani contemporanei, invitando centinaia di autori a depositarvi una propria foto accompagnata da note bio-bibliografiche: nel I93I lo schedario contava più di duecento schede. Di tali richieste rimangono tracce negli epistolari, come nel caso della lettera circolare di ringraziamento segnalata da Ragusa (1997). Ma il Prezzolini di Columbia non si limitò a rispondere alle necessità presenti, mirando piuttosto a creare condizioni propizie a una maggiore diffusione della cultura italiana e, più specificamente, dei libri in italiano. Collaborando con istituzioni come l'Aguilar Branch (succursale della New York Public Library a Little Italy in East Harlem), il Columbia College e la Benjamin Franklin High School (dove studiavano molti giovani italoamericani), la Casa Italiana collaborò all'organizzazione di conferenze settimanali che si tenevano nel centro di assistenza sociale del quartiere, Harlem House, e in biblioteca - l'Aguilar Branch, appunto, dove Prezzolini aveva frequenti contatti con la bibliotecaria Leonilda Sansone, curatrice della sezione italiana del Bulletin of New York Public Library. Sempre nello stesso periodo Prezzolini fondò un «Italian Book of the Month Club» e tentò di avviare corsi di formazione per i librai degli Stati Uniti nord orientali, con lo scopo di informarli sulle "principali collezioni di classici, le case editrici ed il loro carattere, la letteratura moderna, i libri d'arte, le collezioni scientifiche e tecniche, i libri popolari per immigrati, le riviste principali, i giornali e le regole del commercio librario» (Ragusa 1997: 37).

Prezzolini collaborò inoltre a lungo con Andrea Ragusa, editore e libraio di cose italiane in New York sotto l'insegna S.F. Vanni. In effetti, il primo volume stampato da Ragusa nacque proprio da una collaborazione con Prezzolini: una strenna compilata per il centenario della nascita di George Washington. In seguito Prezzolini coinvolse Ragusa come stampatore per un progetto di rivista: l'Italian Literary Digest, periodico di letteratura italiana in traduzione inglese. Dopo la fine della Seconda Guerra Mondiale l'interesse del pubblico statunitense per gli scrittori italiani andava aumentando: Prezzolini immaginava di servire una porzione crescente di interessati a cose italiane che non leggevano l'italiano. Della rivista uscì solo il numero pilota, ma altri progetti editoriali ebbero più fortuna: insieme a Ragusa, Prezzolini pubblicò ancora i risultati dei propri lavori accademici (Prezzolini 1948a, I948b) e creò tre collane di volumi, sia in italiano sia in traduzione inglese, per le quali Vanni pubblicò al ritmo di una decina di titoli l'anno. «Paterno Library Collection of Italian Studies» ospitava studi e tesi di laurea, «Casa Italiana Collection of Italian Classics» presentava traduzioni di classici italiani con testo a fronte (Dante, Guicciardini, Carducci), «Old and New 
Sheaves» offriva invece testi rari, di autori come D'Annunzio, Livingston e Pareto. Fra le note diaristiche si legge quale impresa culturale Prezzolini volesse tentare:

Tutta questa gente [le organizzazioni elettorali degli italoamericani] non sa nulla dei classici; ma sente ogni tanto che gli Americani puri parlano di Dante, di Petrarca, di Boccaccio, di Machiavelli. Facciamogli una collezione dove essi, che non hanno mai saputo nulla di italiano, salvo il dialetto nativo, e dell'inglese non leggono che le notizie di borsa nei giornali, si possano vantare di quei nomi: «Noi siamo della razza di Dante, di Petrarca, di Machiavelli». E aggiungiamo gli artisti, che riprodurremo in belle edizioni. Ciò solleticherà il loro amor proprio. Sottoscriveranno a quella collezione, e metteranno i libri, come si usa in molte case americane, rilegati in pelle e costole dorate, perché il ,padrone' americano sia costretto a riconoscere in loro qualche cosa di più di un coltivatore di carote, di sedani e di zucchini. Ma noi faremo delle buone edizioni, come testo, e delle nuove traduzioni che saranno apprezzate dai dieci o quindici specialisti della letteratura italiana; entreranno allora in tutte le biblioteche (Prezzolini 1980: 99).

Dell'attività di questo periodo l'archivio luganese conserva tracce ancora da studiare, che andranno confrontate con i documenti conservati presso le istituzioni statunitensi.

Dopo la pensione, ormai Professor emeritus, dal 1950 Prezzolini smise di frequentare l'ambiente accademico e condusse vita sempre più ritirata, mentre era attivissimo come giornalista per le testate italiane. Più attivo ancora era il Prezzolini estensore di lettere, come si legge fra le ultime pagine dell'opera autobiografica L'italiano inutile:

Nella rarefazione delle conoscenze "sociali" mi rimasero alcuni antichi amici d'Italia, che consideravo morti come me, sebbene si credessero vivi, e la corrispondenza con loro è come un dialogo d'ombre, carissimo e melanconico, che si prolunga fin d'ora; e poi, a poco alla volta, sempre attraverso il servizio postale, mi feci amici nuovi, che chiamo amici di lettera, non avendoli mai visti, e che apprezzo e ho cari teneramente, come ultime dolcezze della mia penombra (1953: 345).

Nel 1962 Prezzolini rientrò in Europa, dove trascorse gli ultimi vent'anni, prima a Ravello e Vietri sul Mare, poi, dal I968, a Lugano, mantenendo i legami con vecchie e nuove conoscenze attraverso le lettere e le visite che riceveva.

Gli studi sull'«amico di lettera» Goffredo Parise hanno portato a ricerche ulteriori sulla circolazione internazionale dei libri, sulle trasformazioni dell'editoria italiana fra gli anni Cinquanta e Settanta, sulla ricezione delle scoperte scientifiche negli ambienti letterari italiani, oltre a una conoscenza 
più approfondita dell'opera e dell'attività dei due scrittori coinvolti. Nuovi cantieri di lavoro su altri carteggi e documenti del Fondo Prezzolini avranno il pregio di apportare novità in merito a questi e altri temi ancora.

\section{Bibliografia}

Adamson, Walter, «Prezzolini e "La Voce” visti dall'America», Cartevive, III, I (marzo I992), pp. I6-I9.

Bertoni, Brenno - Chiesa, Francesco, Carteggio I9oo-I940, a cura di G. Orelli e D. Rüesch, Lugano, Casagrande, I994.

Biondi, Marino, Giuseppe Prezzolini. Diario di un secolo, Bolzano, Centro di cultura dell'Alto Adige, 200I.

- La cultura di Prezzolini, Firenze, Polistampa, 2005.

Bonini, Tatiana - Callegari Maestretti, Roberta - Rüesch, Diana, «Fondo Giambattista Angioletti: Inventario della corrispondenza», Cartevive, XIV, 2 (34, luglio 2003), pp. I8-63.

Cadioli, Alberto, Letterati editori, Milano, Saggiatore, I995.

Calgari Intra, Fiorenza, Guido Calgari: un uomo e il suo paese, Locarno, Dadò, I990.

Campanile, Marina, Prezzolini, l'intellettuale, "La Voce”, Napoli, Loffredo, 1985.

Campo, Cristina, Lettere a un amico lontano, a cura di A. Spina, Milano, Scheiwiller, I989.

Campo, Cristina - Spina, Alessandro, Carteggio, Brescia, Morcelliana, 2007.

Ceccuti, Cosimo (a cura di), Prezzolini e il suo tempo, Firenze, Le Lettere, 2003.

—. «Prezzolini e Spadolini: l'amicizia di una vita», in Ceccuti 2003, pp. 20I217.

Ceronetti, Guido, La fragilità del pensare, a cura di E. Muratori, Milano, Rizzoli, 2000.

-. Per le strade della Vergine, Milano, Adelphi, 2016.

-. Due cuori e una vigna. Lettere ad Arturo Bersano (I968-I972), con cinque lettere di Arturo Bersano, Padova, Il notes magico, 2017.

Ceronetti, Guido - Quinzio, Sergio, Un tentativo di colmare l'abisso. Lettere I968-I996, a cura di G. Marinangeli, Milano, Adelphi, 20I4.

Chiesa, Francesco - Formiggini, Angelo Fortunato, Carteggio I909-1933, a cura di G. Costa, Bellinzona, Edizioni dello Stato del Cantone Ticino, 2010.

Colla, Enzo, «Giuseppe Prezzolini in America e in Canada», Cartevive, III, 2 (settembre 1992), pp. 3-5.

Costantini, Paola - Rüesch, Diana - Stefanski, Karin, Bibliografia aggiornata delle opere in volume (1903-2007) di Giuseppe Prezzolini, Lugano, Archivio Prezzolini, 2007. 
Cucchiaro, Alex, «Le fotografie dell'Archivio Prezzolini», Cartevive, VII, 2 (novembre I996), pp. 32-37.

Denti di Pirajno, Alberto, Un medico in Africa, Vicenza, Neri Pozza, 1952.

Ferrario, Michele, «Tra lucidità e scetticismo: il terzo Diario di Giuseppe Prezzolini», Cartevive, X, I (23, aprile I999), pp. II-I8.

Ferrario, Michele - Rüesch, Diana, Bibliografia degli scritti di Flaiano, Milano, Scheiwiller, 1988.

Flaiano, Ennio, La notte porta consiglio e altri racconti cinematografici, a cura di D. Rüesch, Milano, Bompiani, 200 .

- Satira è vita. I disegni del Fondo Flaiano della Biblioteca cantonale di Lugano. Con cinquanta brevi testi di Ennio Flaiano, a cura di D. Rüesch, Bologna, Pendragon, 2002.

- Scena all'aperto. Sceneggiatura inedita da una novella di Marino Moretti, a cura di V. Petrocchi, Bologna, Clueb, 2004.

-. La sceneggiatura originale di "Fantasmi a Roma", a cura di A. Pintaldi, Roma, MiBAC, 2007.

-. Un amore a Roma. Dal romanzo al film. Sceneggiatura di Flaiano tratta dal romanzo di Ercole Patti, a cura di F. Natalini, Roma, Artemide, 20 oro.

Flaiano, Rosetta, «Flaiano: i suoi libri, le sue letture, gli anni Trenta e Quaranta», Cartevive, I, 2 (settembre I990), pp. 9-I2.

Gentile, Emilio, «Giuseppe Prezzolini e l'esperienza americana», Cartevive, V, I (marzo I994), pp. 6-I6.

—. «Prezzolini e l'America negli anni del fascismo», in Ceccuti 2003, pp. 233252.

—. «Prezzolini, Giuseppe», in Dizionario Biografico degli Italiani, Roma, Istituto della Enciclopedia italiana, vol. 85, 20I6, ad vocem. Consultabile online al sito: http://www.treccani.it/enciclopedia/giuseppe-prezzolini_\%28Dizionario-Biografico\%29/.

Kouzel Fornacca, Daisy, «Niente "office hours" dal Professor Prezzolini», Cartevive, III, 2 (settembre I992), pp. 24-26.

Luti, Giorgio - Ragusa, Olga - Rüesch, Diana, Giuseppe Prezzolini testimone della sua epoca (I882-I982), Roma, Istituto della Enciclopedia italiana Treccani, I992.

Magherini, Simone, «Prezzolini alla Casa Italiana di Columbia University», Nuova Antologia, I46, 2259 (luglio-settembre 20II), pp. I24-I42.

Marazzi, Martino, Little America, gli Stati Uniti e gli scrittori italiani del Novecento, Milano, Marcos y Marcos, I997.

—. «Prezzolini all'ombra della statua della libertà», Cartevive, X, 2 (24, giugno 1999), pp. I2-22.

Marchione, Margherita, Prezzolini. Un secolo di attività. Lettere inedite e bibliografia di tutte le opere, Milano, Rusconi, 1982. 
Orelli, Giovanni, «Il Fondo Chiesa: tracce per un epistolario», Cartevive, II, I (marzo I99I), pp. II-I2.

Parise, Goffredo, Opere, a cura di B. Callegher e M. Portello, Milano, Mondadori, 200I.

Pellizzato, Giulia, «Goffredo Parise e Giuseppe Prezzolini: un intreccio di relazioni e media», Net(work)s. Entre structure et métaphore / Tra struttura e metafora / Entre estructura y metáfora, a cura di C. Benito Moreno et al., Berlin, Philologie im Netz, 20I6, pp. I08-ı2I.

—. "Litaliano inutilissimo sono io!» Il carteggio tra Parise e Prezzolini: edizione, commento e studio, Tesi di dottorato, Università della Svizzera italiana, A.A. 2018.

Pino Pongolini, Francesca, I cento anni di Giuseppe Prezzolini, Lugano, Biblioteca cantonale, 1982 .

Pino Pongolini, Francesca - Rüesch, Diana, Archivio Prezzolini. Inventario, Bellinzona, Dipartimento della Pubblica Educazione, I989.

Prezzolini, Giuseppe, The legacy of Italy, New York, S.F. Vanni, I948a.

- Repertorio bibliografico della storia e della critica della letteratura italiana dal I933 al I942, New York, S.F. Vanni, I948b.

-. Litaliano inutile, Milano, Longanesi, 1953.

-. Spaghetti Dinner, New York, Abelard-Schuman, I955.

-. Tutta l'America, Firenze, Vallecchi, 1958.

-. Machiavelli, New York, Farrar Straus \& Giroux, I967.

-. Diario I942-I968, Milano, Rusconi, I980.

—. Bruschette «ticinesi», Giubiasco, Edizioni Gottardo, 1983.

Prezzolini, Giuseppe - Papini, Giovanni, Storia di un'amicizia I9oo-I924, Firenze, Vallecchi, 1966.

—. Storia di un'amicizia I925-I956, Firenze, Vallecchi, I968.

Quaretti, Lea, La voce del fiume, Venezia, Neri Pozza, I947.

Quatriglio, Giuseppe, «Il caso della Casa Italiana di Columbia University», Cartevive, IX, I (aprile I998), pp. 23-27.

-. «Il guestbook della Casa Italiana della Columbia University di New York», Cartevive, XIII, I (3I, gennaio 2002), pp. 42-45.

-. «Libri e pensiero di Prezzolini nei Paesi europei e in Giappone», Cartevive, XXII, 46 (agosto 20II), pp. 92-97.

Ragusa, Olga, "Giuseppe Prezzolini professore universitario a New York», in Gabinetto Scientifico Letterario G.P. Vieusseux (a cura di), Giuseppe Prezzolini testimone della sua epoca (I882-I982), Roma, Istituto della Enciclopedia italiana Treccani, I992, pp. 34-50.

—. "Quando gli archivi parlano», in S. Betocchi (a cura di), Giuseppe Prezzolini, The American Years 1929-I962, New York-Firenze, S.F. Vanni - Gabinetto G.P. Vieusseux, I994, pp. 39-62. 
—. «Prezzolini e la diffusione del libro italiano», Cartevive, VIII, I (aprile 1997), pp. 34-37.

-. Gli anni americani di Giuseppe Prezzolini, Firenze, Le Monnier, $200 \mathrm{I}$.

Rebay, Luciano, «Prezzolini e gli Stati Uniti», in Ceccuti 2003, pp. 22I-232.

Rüesch, Diana, «Importanza e contenuti del Fondo Flaiano di Lugano», Cartevive, I, 2 (settembre 1990), pp. 4-6.

—. «L'Archivio Prezzolini e gli Archivi di Cultura Contemporanea. Un viaggio attraverso le carte», Cartevive, VIII, I (aprile 1997), pp. 3-6.

-. «Testimonianze cartacee del Novecento italiano e svizzero alla Biblioteca cantonale di Lugano: fondi, strumenti, attività, pubblicazioni», Archivio Storico Ticinese, seconda serie, $\mathrm{I} 30$ (dicembre 200I), Appendice, pp. I-20.

—. «Le carte ancora sconosciute dell'Archivio Prezzolini conservato alla Biblioteca cantonale di Lugano», in Ceccuti 2003, pp.303-317.

—. «L'archivio Prezzolini», in A. Ria (a cura di), Progetto Biblioteca. Spazio, storia e funzione della Biblioteca cantonale di Lugano, Losone-Lugano, Edizioni Le Ricerche, 2005, pp. I25-136.

-. (a cura di), Dal Fondo senza fondo. 50 testimonianze per Guido Ceronetti, numero monografico di Cartevive, XVII, I (40, agosto 2007).

—. «Mario Agliati e Giuseppe Prezzolini. Sotto il segno di una vicendevole stima», Il Cantonetto, 2012, pp. 50-57.

Rüesch, Diana - Franciolli, Marco, Dalla buca del tempo: la cartolina racconta. I collages di cartoline d'epoca del Fondo Guido Ceronetti, Lugano, Biblioteca cantonale - Archivi di Cultura italiana - Museo Cantonale d'Arte, 2000.

Rüesch, Diana - Stefanski, Karin, «Uno che ha tempo: Ennio Flaiano sceneggiatore. Catalogo della mostra», Cartevive, XXI, 45 (novembre 20I0), pp.3-94.

-. «Per le strade» di Guido Ceronetti. Omaggio allo scrittore per $i$ suoi 90 anni, numero monografico di Cartevive, XXVIII, 56 (dicembre 20I7).

Salek, Roberto, Prezzolini. Una biografia intellettuale, Firenze, Le Lettere, 2002. Saltini, Luca, Il viaggiatore della parola: G.B. Angioletti (I896-I96I), Lugano Losone, Biblioteca cantonale di Lugano - Edizioni Le Ricerche, 2007.

Simonetti, Carlo Maria, "Giuseppe Prezzolini "impresario di cultura" , Inventario, nuova serie, IXI, 2 (maggio-agosto I98I), pp. I25-I26.

Stefanski, Karin, «Visita guidata virtuale alla mostra dedicata a Fulvio Tomizza nel decennale della scomparsa», Cartevive, XX, 2 (44, dicembre 2009), pp. 6-20.

Vignoli, Francesco, «Prezzolini e "Il Borghese" di Longanesi ossia l'ultima avventura giovanile della sua vita», Cartevive, VII, 2 (novembre 1996), pp. 39-4I. 


\section{Indice dei manoscritti}

Lugano

Biblioteca cantonale

Archivio Prezzolini

Fondo Giuseppe Prezzolini

Corrispondenze "antiche" (I900-1945)

Corrispondenze "moderne" (I946-I978)

Raccolta degli articoli di Prezzolini

Raccolta di recensioni alle opere di Prezzolini

Raccolta di articoli su Prezzolini e l'ambiente vociano

Fondo Alessandro Spina

Fondo Fulvio Tomizza

Fondo Giovanni Battista Angioletti

Fondo Enrico Emanuelli

Fondo Guido Ceronetti

Fondo Guido Calgari

Fondo Ennio Flaiano

Fondo Francesco Chiesa

New York

New York Public Library

Farrar, Straus \& Giroux records

Pavia

Università degli Studi

Centro Manoscritti - Centro per gli studi sulla tradizione manoscritta di autori moderni e contemporanei

Fondo Ennio Flaiano

Vicenza

Biblioteca Bertoliana

Fondo Neri Pozza

Corrispondenze Autori, fascicolo Denti di Pirajno, Lettera di Neri Pozza ad Alberto Denti di Pirajno (or.04.I953).

Archivio Scrittori Vicentini del Novecento 